\title{
Backflow-Induced Asymmetric Collapse of Disclination Lines in Liquid Crystals
}

\author{
Patrick Oswald* and Jordi Ignés-Mullol ${ }^{\dagger}$ \\ Laboratoire de Physique, Ecole Normale Supérieure de Lyon, 46 allée d'Italie, 69364 Lyon, Cedex 07, France
}

(Received 13 December 2004; published 6 July 2005)

\begin{abstract}
We present experiments where opposed pairs of planar parallel disclination lines of topological strength $s= \pm 1$ move due to their mutual attraction. Our measurements show that their motion is clearly asymmetric, with +1 defects moving up to twice as fast as -1 ones. This is a clear indication of backflow, given the intrinsic isotropic elasticity of our system. A phenomenological model is able to account for the experimental observations by renormalizing the orientational diffusivity estimated from the velocity of each defect.
\end{abstract}

DOI: 10.1103/PhysRevLett.95.027801

PACS numbers: 61.30.Jf, 61.30.Hn, 61.72.Lk, 83.80.Xz

Topological defects were first postulated in order to explain the plasticity of metals. Then, they were proved to be essential for understanding phase transitions, disordered systems, frustrated media, convective instabilities, as well as some biological shapes, etc. In real systems, defects interact, leading to complex dynamics that often result in the annihilation of pairs of defects of opposite signs. Such phenomena arise, for instance, during solid recrystallization, but they can similarly be observed in liquid crystals where they are much easier to study experimentally.

In nematic (or cholesteric) liquid crystals, defects are disclination lines that break locally the orientational order. Their motion induces a distortion of the director field, starting at the defect core and propagating diffusively in the bulk. Early analysis assumed that the dynamical director field was the same as under static conditions at all times [1]. This quasistatic approximation leads to a logarithmic divergence of the calculated total dissipated energy, which requires one to introduce a cutoff length at large distances from the core of the defect. Ryskin and Kremenetsky [2] later showed that this characteristic length comes out naturally from the dynamic equations and is of the order of the orientational diffusivity divided by the velocity of the defect. This length defines an active zone around the defect in which the quasistatic approximation is valid. On the other hand, liquid crystals flow as liquids. One thus may expect that defect motion, which is mainly due to an inplace rotation of the director, is affected by hydrodynamics (the so-called backflow effects). Recent numerical simulation in two dimensions [3] of the annihilation of two planar disclination lines of strengths $s= \pm 1 / 2$ in nematics have confirmed this point. It was found numerically that backflow breaks the symmetry of the collapse dynamics between defects, even in isotropic elasticity, the $+1 / 2$ defect moving faster than the $-1 / 2$ one. A similar conclusion was reached in simulations for the collapse of \pm 1 disclinations in smectic- $C$ films [4]. These results are consistent with the analysis by Kats et al. [5] who found that positive disclinations always exert a stronger influence on the flow velocity than negative ones.
There has been some experimental work dealing with the interaction between parallel disclination lines. Most of it has involved statistical studies, either of the evolution of disclination lines as annihilation of pairs took place [6] or of the coarsening dynamics of defect strings and loops [7]. Recent experiments [8] have addressed directly the dynamics of the annihilation of parallel $\pm 1 / 2$ disclination lines in a planar nematic sample. In that case, the dynamics is strongly disturbed by the interactions between the defects and the glass plates, which could explain why no asymmetry was mentioned.

In this Letter, we present clear experimental evidence of the asymmetric motion of two $s=+1$ and $s=-1$ parallel planar disclination lines attributable to backflow effects. These occur mainly in the vicinity of the core of the defects, whose size can be continuously tuned with an external electric field. Moreover, we propose to interpret our experimental results in the framework of a semiphenomenological model assuming that the director field around the defects is the same as in the absence of motion, whereas two different effective rotational diffusivities are attributed to each defect for taking into account backflow effects.

In order to succeed at these experiments, the defect motion must be decoupled from the anchoring conditions imposed on the cell plates. Moreover, isotropic elasticity is desirable to rule out an elastic origin in any detected motion asymmetry. In our experiments, rectangular cells $2 \times 2 \mathrm{~cm}^{2}$ of indium-tin-oxide-coated glass are assembled with a spacing $d=116 \mu \mathrm{m}$ between them (using nylon threads). The inner surface is treated to achieve homeotropic anchoring with the silane product ZLI-3124 (Merck). An alternative surface treatment with N,N-dimethyl-N-octadecyl-3-aminopropyl trimethoxysilyl chloride (DMOAP) using the protocol described by Kahn [9] has also been used for comparison. The cholesteric mixture is prepared using the nematic liquid crystal ZLI 2806 (Merck) and $0.52 \mathrm{wt} \%$ of the chiral dopant ZLI 811 (Merck). The material has a negative dielectric anisotropy 
$\epsilon_{a}=-4.8$ at $f=1 \mathrm{kHz}$ and a cholesteric pitch $p=$ $23.8 \mu \mathrm{m}$.

Abrupt application of a strong electric field imposes the planar orientation of the molecules through most of the sample thickness, with many disclination lines nucleating perpendicular to the electrodes [6]. Because the lines can freely glide over the homeotropic boundary layers at the plates (layer thickness is inversely proportional to the electric field), the anchoring neither disturbs the line dynamics nor imposes any preferred direction of motion (gliding anchoring). On the other hand, the condition of isotropic elasticity (not fulfilled in general because splay and bend elastic constants $K_{1}$ and $K_{3}$ are often different in nematics) is automatically satisfied in our cholesteric system, where the director winds around the axis perpendicular to the electrodes. Let $\vec{c}$ be the projection of the director in the middle plane. It can be verified that, provided the cholesteric pitch $p$ is much smaller than the sample thickness $d$, the system will effectively behave as a nematic layer with equal bend and splay elastic constants $K=$ $\left(K_{1}+K_{3}\right) / 2$. As $\vec{c}$ and $-\vec{c}$ are not equivalent, only \pm 1 disclination lines will form (spinlike system).

Cells are filled by capillarity with the mixture, and experiments are performed at room temperature. Upon filling, the cell is populated with disordered threadlike defects, which may be attached to the plates (on dust particles or inhomogeneities of the anchoring) and do not disappear spontaneously. In order to prepare suitable initial conditions, free from defects, the sample is annealed overnight applying an ac voltage $V_{\text {rms }}=2 \mathrm{~V}, f=2 \mathrm{~Hz}$, which triggers weak electrohydrodynamic convection. Next, $f$ is increased to $1 \mathrm{kHz}$ and $V$ to $10 \mathrm{~V}$, leading to a sample with only bulk disclination lines, i.e., detached from the plates. Such disclinations eventually annihilate, creating regions of several $\mathrm{mm}^{2}$ with a uniform planar cholesteric texture (i.e., with cholesteric "layers" parallel to the electrodes).

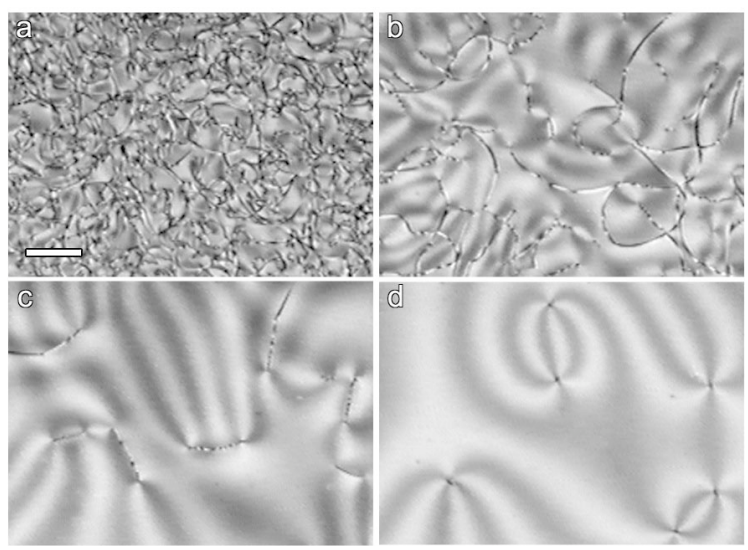

FIG. 1. Nonparallel disclination lines formed after the electrohydrodynamic instability triggered at $17 \mathrm{~V}$ and $1 \mathrm{~Hz}$ disappear under higher frequency conditions $(80 \mathrm{~V}, 1 \mathrm{kHz})$. The line segment in (a) is $100 \mu \mathrm{m}$ long. Total elapsed time is $100 \mathrm{~s}$.
The parallel disclination lines that are the object of our study are created by triggering an electrohydrodynamic instability for a few seconds (by lowering $f$ to about $1 \mathrm{~Hz}$ ) and subsequently setting the desired value for $V$ at $f=1 \mathrm{kHz}$. The sample is initially populated by nonparallel disclination lines [Fig. 1(a)] but, after a short transient (about $1 \mathrm{~min}$ ), only a scarce number of parallel disclination lines of topological charges \pm 1 , perpendicular to the cell plates, survive. The subsequent evolution consists in the progressive annihilation of neighboring lines of opposed signs [Fig. 1(d)]. For our study, we monitor the motion of interacting defect pairs whose initial separation is less than one-third the distance to the next nearest defect. Nonnegligible interactions with other defects result in nonrectilinear trajectories (that are discarded), limiting the range of our usual observations to, typically, distances smaller than the sample thickness. We have measured the trajectory of defect pairs as a function of the applied voltage across the electrodes. Observation is performed in a polarizing microscope with crossed polarizers. Images from a CCD camera are stored in a time-lapse VCR and digitized for further analysis.

Asymmetry in the defect motion has been observed in all the experimental realizations, with +1 defects moving faster. In Fig. 2, defect trajectories for a series of experiments under the same applied voltage are shown. The asymmetric motion is clearly seen by comparing the meeting point, which is not halfway between the initial positions of the defects. Asymmetry and, therefore, backflow effects, are more evident as defects approach the collapse, with the +1 defect reaching a maximum speed about twice that of the -1 , although these effects are non-negligible even at velocities lower than $0.1 \mu \mathrm{m} \mathrm{s}^{-1}$. The ratio between the speed of the two defects changes over time, increasing as defects approach.

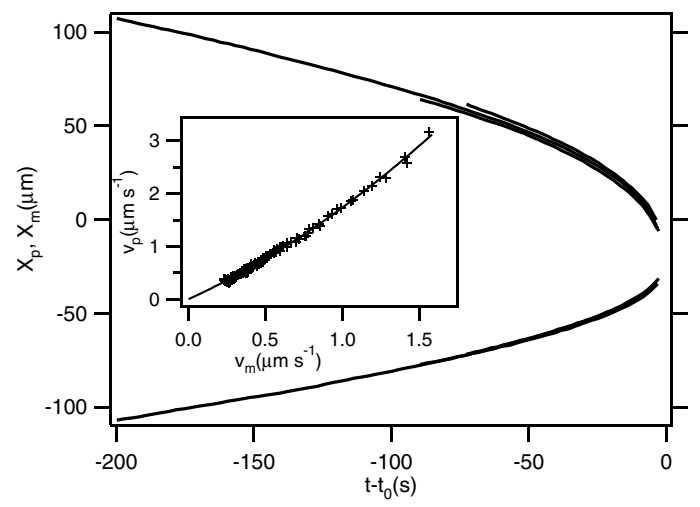

FIG. 2. Motion of three series of defects towards their annihilation under an applied tension of $60 \mathrm{~V}$. Defects move along the $x$ axis. The position of the $s=+1$ defects $(p)$ is positive. The reference is set halfway between the defects when measurements start. In the inset, data show the relative speeds. The solid line is a parabolic fit, illustrating that asymmetry increases as defects approach. 
Next, we show that backflow effects can be effectively described by incorporating a renormalized rotational viscosity in the viscous force and a renormalized elastic constant in the elastic force. The analysis is greatly simplified because of isotropic elasticity, which in the present system is an almost exact result [10].

In the quasistatic approximation, the structure of the director field in the presence of moving planar disclination lines is assumed to be, at all times, the same as at rest. It can be calculated everywhere, in particular, inside the core of each line where the director aligns along the disclination axis to avoid the formation of a singularity. Moreover, it is assumed that the motion of the lines results in simple inplace rotation of the director. With this, the rate of dissipated energy, $\Phi$, can be written as

$$
\Phi \equiv 2 F_{v} U,
$$

where $U$ is the (symmetric) defect velocity. Straightforward calculation of $\Phi$ in the quasistatic limit leads to the following expression for the viscous force:

$$
F_{v}=\pi \gamma_{1} U\left[\ln \frac{R}{r_{c}}+\frac{1}{2} \ln \frac{(\xi / 2)^{2}+R^{2}}{(\xi / 2)^{2}+r_{c}^{2}}+\frac{\pi^{2}}{6}\right] .
$$

Here, $\gamma_{1}$ is the rotational viscosity, $r_{c}$ is the core radius, $\pi^{2} / 6$ results from the dissipation inside the core, $\xi$ is the distance between defects, and $R$ is a long range cutoff length marking the region around the defect where dissipation is significant. It is far from being obvious how to measure the core radius. Experimentally, it appears as a black spot whose size depends on the focus settings and increases for lower applied voltages. For that reason, we use the calculated theoretical value for $r_{c}$. It was obtained by considering a helicoidal profile for the director field around the core, with the polar tilt angle $\theta(r)$ being a function of the distance $r$ from the disclination axis (thus invariant along the line). Since the electric field is parallel to the line, $\theta=\pi / 2$ away from the disclination and vanishes at $r=0$. Straightforward calculation of the total free energy and its minimization lead, within a good approximation, to the following expression for $\theta(r)$, valid for both +1 and -1 defects:

$$
\theta(r)=\frac{\pi}{2} \frac{r^{2}}{r^{2}+r_{c}^{2}}
$$

In this equation $r_{c}$ is the core radius given by

$$
r_{c}=\frac{\sqrt{2}}{\sqrt{q_{o}^{2}+\xi_{E}^{-2}}},
$$

with $q_{o}=2 \pi / p$ the spontaneous twist and $\xi_{E}=$ $\sqrt{K /\left(\epsilon_{o}\left|\epsilon_{a}\right|\right)}(d / V)$ the electric coherence length (which, additionally, gives the thickness of the two boundary layers in which the director rotates by $\pi / 2$ close to the electrodes). Using values for the elastic constants and the dielectric anisotropy $\epsilon_{a}$ given by Merck, this formula becomes

$$
r_{c}=\frac{5.0 \mu \mathrm{m}}{\sqrt{1+3.1 \times 10^{-3} V^{2}}} .
$$

The speed of the defects is determined by balancing viscous and elastic forces, $F_{e}=2 \pi K / \xi$, which gives

$$
U=\left(2 K / \gamma_{1}\right) \xi^{-1} f(\xi ; U)^{-1},
$$

where $f(\xi ; U)$ is the expression between square brackets in Eq. (2). Notice that $U$ enters through the cutoff length $R$.

Even though the above expressions are valid only in the absence of backflow (symmetric motion), we use them as an ansatz to analyze our experimental data. In particular, we use Eqs. (2) and (6) for calculating separately the velocity of each defect, by assuming different values $D_{p}=$ $K_{\text {eff }} / \gamma_{1}^{p}$ and $D_{m}=K_{\text {eff }} / \gamma_{1}^{m}$ for the effective rotational diffusivity $D_{\text {eff }}$ associated with defects +1 and -1 . As for the cutoff length $R$ associated with each defect, we let $R=(3.6 K) /\left(\gamma_{1} v_{p, m}\right)$ [that way, Eq. (2) gives back, in the limit $\xi \rightarrow \infty$, the formula given by Ryskin and Kremenetsky for an isolated defect]. It is calculated by taking the measured defect velocity and the value of the static rotational diffusivity $K / \gamma_{1}\left(K=1.5 \times 10^{-6}\right.$ dyn, $\gamma_{1}=2.4$ poise), since backflow effects are negligible at long distances from the defects. With this protocol, $D_{\text {eff }}$ is obtained for defects of either sign for each applied voltage (Fig. 3). Notice that the linearity predicted by the symmetric calculation in Eq. (6) is still reasonably preserved in the presence of backflow. Although not constant, $D_{\text {eff }}$ varies very little in the full range of measured velocities. Of course, the above analysis assumes that each defect lies inside the active zone (previously defined) of the other defect. That means that the cutoff length $R$ defined above must be significantly larger than the defect distance. We

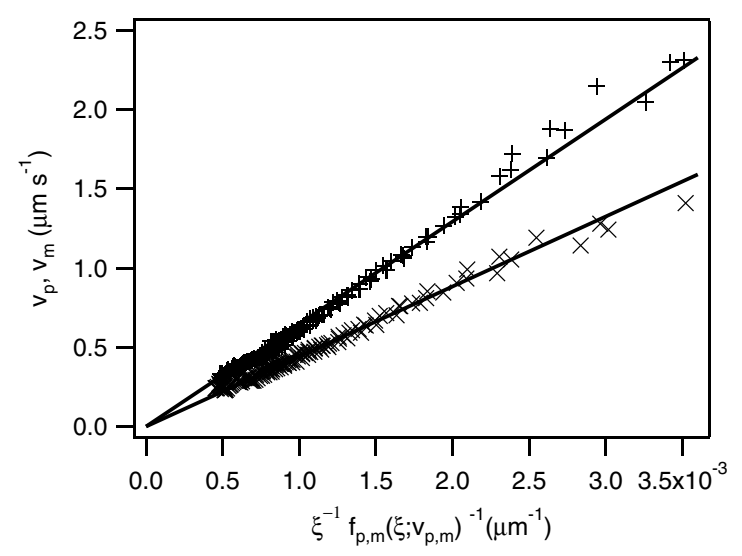

FIG. 3. Instantaneous defect speed of positive $(p,+)$ and negative $(n, \times)$ defects for the three independent data sets shown in Fig. 2 plotted using the functional form in Eq. (6) with a value for $R$ that depends on the actual defect velocity. The slope of each curve yields the effective rotational diffusivity of each defect. 


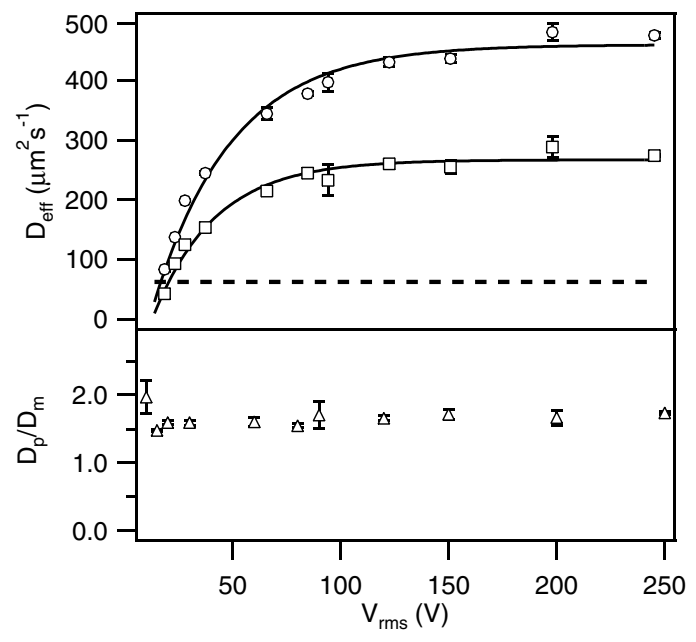

FIG. 4. Effective rotational diffusivity $(K / \gamma)$ for moving disclination lines of opposed topological charges ( $\square$ is $-1, \bigcirc$ is $+1)$ as a function of the applied voltage, obtained as detailed in the text. Their ratio is shown in the bottom graph. The solid lines are guides for the eye. The dashed line marks the static rotational diffusivity for this compound.

have verified that this is, indeed, the case in the analyzed data ( $R$ is always at least 3 times larger than $\xi$ ). The result of this analysis for the full range of voltages explored is presented in Fig. 4, where data obtained for samples prepared with the two alternative surface treatments are combined, showing that anchoring effects can, indeed, be neglected. This is true except at high applied voltage, where homeotropic anchoring starts to fail [11]. This is evidenced by defects being pinned on the plates and by an anomalous deceleration of the lines. Measurements with the DMOAP treatment are possible for voltages as high as $250 \mathrm{~V}$, while those with the Merck silane fail beyond $150 \mathrm{~V}$. At the opposite end of the range of applied voltages (below $5 \mathrm{~V}$ ), the planar configuration of the director field becomes unstable. At these voltages, defects are motionless. A possible explanation is that they are blocked by the undulations of the two boundary layers.

To conclude, our experiments clearly show that the collapse dynamics of two defects of strengths \pm 1 is strongly asymmetric. This effect must be attributed to backflow effects and not to elastic anisotropy, which can be ruled out in our system because of its helical structure, allowing us to replace the elastic constants $K_{1}$ and $K_{3}$ by their arithmetical mean. A phenomenological model allows us to reproduce at each voltage the collapse dynamics and its asymmetry, with the condition that we attribute to each defect a different effective rotational diffusivity. In practice, these two values are larger than the intrinsic rotational diffusivity of the material [12], which is consistent with existing experimental observations $[13,14]$. In addition, backflow effects are found to be more important for +1 defects than for -1 , resulting in a larger effective diffusivity for the former than for the latter. This is consistent with existing theoretical work on related systems. One could question about the observed voltage dependence of the diffusivities. Our data show than the larger the core radius, the closer the effective diffusivity is to the static value. This result strongly suggests that backflow effects are localized close to the cores of the two defects and do not vary with the voltage when their radii are sufficiently small (high applied voltage). In our experiment, the saturation is observed at voltages larger than $100 \mathrm{~V}$, when the core radii become very small $\left(r_{c}<1500 \AA\right)$. On the contrary, molecules orient homeotropically over a large surface area inside the core of the defects at small voltages, which certainly inhibits backflow effects and explains a smaller effective diffusivity.

The authors acknowledge support from a France-Spain Integrated Action (Picasso program). J. I-M. acknowledges support from the RyC Program from MCyT. We thank E. I. Kats for a critical reading of the manuscript.

*Electronic address: patrick.oswald@ens-lyon.fr

†Permanent address: Departament de Química Física, Universitat de Barcelona, Martí i Franquès 1, 08028 Barcelona, Spain.

[1] H. Imura and K. Okano, Phys. Lett. 42A, 403 (1973).

[2] G. Ryskin and M. Kremenetsky, Phys. Rev. Lett. 67, 1574 (1991).

[3] G. Toth, C. Denniston, and J. M. Yeomans, Phys. Rev. Lett. 88, 105504 (2002); Phys. Rev. E 67, 051705 (2003).

[4] D. Svensek and S. Zumer, Phys. Rev. Lett. 90, 155501 (2003).

[5] E. I. Kats, V. Lebedev, and S. V. Malinin, J. Exp. Theor. Phys. 95, 714 (2002).

[6] T. Nagaya, H. Hotta, H. Orihara, and Y. Ishibashi, J. Phys. Soc. Jpn. 61, 3511 (1992).

[7] I. Chuang, B. Yurke, A. N. Pargellis, and N. Turok, Phys. Rev. E 47, 3343 (1993).

[8] A. Bogi, P. Martinot-Lagarde, I. Dozov, and M. Nobili, Phys. Rev. Lett. 89, 225501 (2002).

[9] F. J. Kahn, Appl. Phys. Lett. 22, 386 (1973).

[10] Boundary layers are much thinner than the sample thickness and the number of cholesteric layers is large: about 10 in our experiment.

[11] G. Barbero, L. R. Evangelista, and N. V. Madhusudana, Eur. Phys. J. B 1, 327 (1998).

[12] Except at very small voltages where the motion of the defects is blocked because of a destabilization of the cholesteric layer.

[13] A. Mertelj and M. Copic, Phys. Rev. E 69, 021711 (2004).

[14] P. E. Cladis, W. van Saarloos, P. L. Finn, and A. R. Kortan, Phys. Rev. Lett. 58, 222 (1987). 